\title{
Valuing the Ocean Carbon Sink in Light of National Climate Action Plans
}

By Johannes Karstensen, Wilfried Rickels, Pierre Testor, and Maciej Telszewski

Signatory states to the Paris Agreement are required to formulate and implement national climate action (NCA) plans to direct their nationally determined contributions (NDCs) in reducing greenhouse gases (GHGs) and in particular carbon dioxide $\left(\mathrm{CO}_{2}\right)$ emissions (UNFCCC, 2015). The NCA plans address $\mathrm{CO}_{2}$ management for all components of society, be it food, mobility, energy, or consumption. The extent to which NCA plans are in line with the overall targets will be assessed during global stocktakings, with the first one scheduled for 2023. Limiting global temperature increase by reducing $\mathrm{GHG}$ and $\mathrm{CO}_{2}$ emissions will depend crucially on natural, non-anthropogenic sink efficiency. The ocean currently removes $25 \%-30 \%$ of the $\mathrm{CO}_{2}$ emitted to the atmosphere by human activities (Friedlingstein et al., 2020), thereby providing, alongside other critical ecosystem services, an important societal wealth contribution via $\mathrm{CO}_{2}$ sequestration.

From the above results, it is obvious that the ocean $\mathrm{CO}_{2}$ monitoring system must be able to determine the present and future $\mathrm{CO}_{2}$ uptake with sufficient accuracy. To justify the effort involved, it is also useful to determine the monetary value of the oceanic sink. This can be done in three ways: a cost-benefit analysis (CBA), a cost-effectiveness analysis (CEA), or a market-based $\mathrm{CO}_{2}$ pricing approach. CBA and CEA both use shadow prices in their calculations that measure the social costs of emitting a marginal tonne of carbon ${ }^{1}$, whereas market prices are used in national accounting to compute, for example, the gross domestic product. In CBA, shadow prices are derived from estimates of costs of climate change impacts. In CEA, shadow prices are derived under a given target, such as a temperature

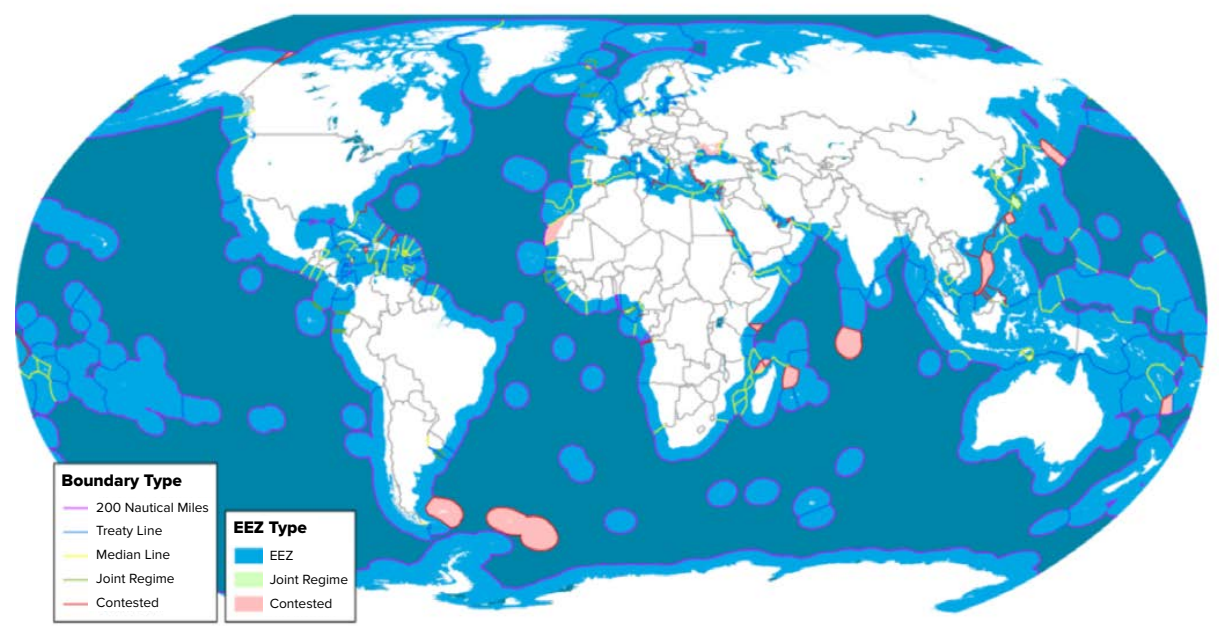

FIGURE 1. World ocean territorial boundaries and Exclusive Economic Zones (EEZs). target as defined in the Paris Agreement. Put simply, CBA provides information on how much wealth is generated by the ocean in terms of reducing climate change, and CEA provides information on how much wealth is generated by reducing emissions abatement costs for the given target. The latter information is in most cases more reliable and can also be obtained with a market-based approach, given the regulatory framework and that a market is in place. Observed $\mathrm{CO}_{2}$ prices can also be used to assess the value of $\mathrm{CO}_{2}$ sinks, even though these sinks are not involved in trading. The market and the underlying regulatory framework are ideally designed to regulate anthropogenic activities, so given natural $\mathrm{CO}_{2}$ sequestration, targets like net-zero $\mathrm{CO}_{2}$ can be achieved. However, a weakening of natural sinks implies, in turn, that faster reductions and additional atmospheric $\mathrm{CO}_{2}$ removal will be required. Similarly, the market price would increase given that a weakening of $\mathrm{CO}_{2}$ sinks is expected to be considered in the underlying regulatory framework, defining the scarcity in a given $\mathrm{CO}_{2}$ market.

Hence, highly reliable information about current and future ocean $\mathrm{CO}_{2}$ sinks provides value information for policymakers to properly align the regulatory framework, and for the business community to form realistic $\mathrm{CO}_{2}$ price expectations. Furthermore, $\mathrm{CO}_{2}$ sequestration by ocean sinks varies regionally-and $38 \%$ of the global ocean comprises territorial waters (Figure 1). Considering the regional variations in $\mathrm{CO}_{2}$ emissions and in sink activity implies that the $\mathrm{CO}_{2}$ wealth effects vary considerably for different countries (Bertram et al., 2021). Although regional differences in terrestrial $\mathrm{CO}_{2}$ sinks are considered in national emissions inventories, regional ocean sink contributions are not yet included in the determination of international burden sharing regarding $\mathrm{CO}_{2}$ emissions abatement. Thus, the current framework favors countries with large terrestrial $\mathrm{CO}_{2}$ sinks to the disadvantage of countries with large territorial ocean sinks. However, costs of ocean $\mathrm{CO}_{2}$ fluxes are highly interconnected: variability in the global ocean $\mathrm{CO}_{2}$ uptake of atmospheric $\mathrm{CO}_{2}$ will influence the $\mathrm{CO}_{2}$ uptake in territorial waters.

Marginal cost of the impacts caused by emitting one extra tonne of carbon dioxide at any point in time. 
Therefore, improved and reliable estimates of ocean $\mathrm{CO}_{2}$ uptake are required to properly steer the ambitious levels included in the NDCs, to allow for improved burden sharing, to attribute territorial ocean $\mathrm{CO}_{2}$ uptake appropriately to countries, and to account for ocean carbon dioxide removal (CDR). Current scenarios still focus predominantly on terrestrial $C D R$, mostly because economic integrated assessment models are not yet capable of properly modeling ocean CDR. Given an increasing requirement for CDR due to the weakening of $\mathrm{CO}_{2}$ sinks, the necessary net $\mathrm{CO}_{2}$ emissions path is unlikely to be achievable without ocean CDR solutions, for example, ocean alkalinity management. However, properly assessing CDR via ocean-based measurements, which will be part of future NDCs, requires accounting for the feedback of territorial ocean $\mathrm{CO}_{2}$ uptake on global $\mathrm{CO}_{2}$ uptake.

In general, the assessment of ocean $\mathrm{CO}_{2}$ sequestration must be based on understanding the processes of and monitoring both fluxes and storage of $\mathrm{CO}_{2}$ in the oceanfrom regional to global scales. The processes controlling $\mathrm{CO}_{2}$ in the ocean are often separated into "solubility pump" (controlled by physics and biogeochemistry) and "biological pump" (controlled by biochemistry and biology) concepts. However, these two pumps are interlinked via underlying processes and may also counteract each other in the matter of net $\mathrm{CO}_{2}$ uptake; for example, in highly productive upwelling regions, the $\mathrm{CO}_{2}$ sink created by the biological pump may compensate for the outgassing of $\mathrm{CO}_{2}$ driven by the solubility pump (Figure 2). Carbon assessments require determining the efficiency of both pumps and coordination of the underlying ocean observations that make use of multiple platforms (ships, underwater as well as surface autonomous vehicles, floats, moorings, and satellites) equipped with sensors and samplers employing a diverse pool of sensing technology (optical, particle probes, electrochemical) and operating at varying levels of technological readiness.

Data harmonization and quality control along with FAIR (Findable, Accessible, Interoperable and Reusable) access to data permit a wide spectrum of applications across disciplines and needs, and they must be ensured to enable integration of the various data streams into regional and global carbon products. Regional (and global) carbon data products with sufficient temporal and spatial resolutions are required for assigning values to Exclusive Economic Zone $\mathrm{CO}_{2}$ sinks. Global coordination of observational efforts, science approaches, and coordination with global syntheses are performed under the auspices of the International Ocean Carbon Coordination Project (IOCCP).
A key aspect for use of data in the context of NCA plans is the global harmonization of error/uncertainties estimates that, for the ocean interior, are based on Reference Material (RM). In order to integrate the benefits of RM into a multiplatform observing system, a reference grid of longterm, sustained observations made using RM needs to be maintained. That in turn is used as a reference for secondary quality control of observations that do not allow direct RM traceability (e.g., expendable sensors or devices with exceptionally long endurance under harsh conditions and impacted by biofouling). IOCCP works directly with the Surface Ocean $\mathrm{CO}_{2}$ NETwork (SOCONET) and the Global Ocean Ship-based Hydrographic Investigations Program (GO-SHIP) to assure global coordination related to data quality standards and protocols. At a regional scale, the station labeling procedure of the Ocean Thematic Center (OTC) of the European Research Infrastructure Integrated Carbon Observation System-Ocean Thematic Centre (ICOSOTC) is a prime example of successful implementation of data management procedures that result in delivery of high-quality information with known uncertainty. ICOSOTC also focuses on other greenhouse gases, and the OTC maintains thematic centers to coordinate atmospheric and terrestrial domains to deliver sustained, truly integrated observations that benefit a variety of stakeholders.

\section{REFERENCES}

Bertram, C., M. Quaas, T.B.H. Reusch, A.T. Vafeidis, C. Wolff, and W. Rickels. 2021. The blue carbon wealth of nations. Nature Climate Change 11:704-709, https://doi.org/10.1038/s41558-021-01089-4. Friedlingstein, P., M. O'Sullivan, M.W. Jones, R.M. Andrew, J. Hauck, A. Olsen, G.P. Peters, W. Peters, J. Pongratz, S. Sitch, and others. 2020. Global carbon budget 2020. Earth System Science Data 12:3,269-3,340, https://doi.org/10.5194/essd-12-3269-2020.

UNFCCC (United Nations Framework Convention on Climate Change). 2015. Adoption of the Paris Agreement, $21^{\text {st }}$ Conference of the Parties, United Nations, Paris.

ARTICLE DOI: https://doi.org/10.5670/oceanog.2021.supplement.02-21 


\section{AUTHORS}

Johannes Karstensen (jkarstensen@geomar.de), GEOMAR Helmholtz Centre for Ocean Research Kiel, Germany. Wilfried Rickels, Kiel Institute for the World Economy, Germany. Pierre Testor, CNRS - Sorbonne Université, Laboratoire d'Océanographie et de Climatologie, Institut Pierre Simon Laplace, Observatoire Ecce Terra, France. Maciej Telszewski, International Ocean Carbon Coordination Project, Institute of Oceanology, Polish Academy of Sciences, Poland.

\section{ARTICLE CITATION}

Karstensen, J., W. Rickels, P. Testor, and M. Telszewski. 2021. Valuing the ocean carbon sink in light of national climate action plans. Pp. 50-51 in Frontiers in Ocean Observing: Documenting Ecosystems, Understanding Environmental Changes, Forecasting Hazards. E.S. Kappel, S.K. Juniper, S. Seeyave, E. Smith, and M. Visbeck, eds, A Supplement to Oceanography 34(4), https://doi.org/10.5670/oceanog.2021. supplement.02-21.

\section{COPYRIGHT \& USAGE}

This is an open access article made available under the terms of the Creative Commons Attribution 4.0 International License (https://creativecommons.org/ licenses/by/4.0/), which permits use, sharing, adaptation, distribution, and reproduction in any medium or format as long as users cite the materials appropriately, provide a link to the Creative Commons license, and indicate the changes that were made to the original content. 